\title{
Preparation of Ferricyanic Acid Doped Aryl Amine Copolymer and Its Electrochemical Properties
}

\author{
Lihuan Xu*, Jiaojiao Ma, Yue Sun, Pengju Guo, Bing Han, Guosheng Wang, Chang Su* \\ College of Chemical Engineering, Shenyang University of Chemical Technology, Shenyang, China \\ Email address: \\ xulihuanss@163.com (Lihuan Xu), suchang@syuct.edu.cn (Chang Su) \\ ${ }^{*}$ Corresponding author

\section{To cite this article:} \\ Lihuan Xu, Jiaojiao Ma, Yue Sun, Pengju Guo, Bing Han, Guosheng Wang, Chang Su. Preparation of Ferricyanic Acid Doped Aryl Amine \\ Copolymer and Its Electrochemical Properties. Science Discovery. Vol. 6, No. 4, 2018, pp. 276-282. doi: 10.11648/j.sd.20180604.18
}

Received: June 30, 2018; Accepted: August 1, 2018; Published: August 10, 2018

\begin{abstract}
In this paper, five hybrid copolymers of $\mathrm{HCF} / \mathrm{PANI}, \mathrm{HCF} / \mathrm{P}(\mathrm{ANI}: \mathrm{pPD}=5: 1), \mathrm{HCF} / \mathrm{P}(\mathrm{ANI}$ :pPD=3:1), $\mathrm{HCF} / \mathrm{P}(\mathrm{ANI}: \mathrm{pPD}=1: 1), \mathrm{HCF} / \mathrm{PpPD}$ were prepared by in-situ synthesis using the prepared ferricyanic acid (HCF) as doping acid with electrochemical activity. The prepared hybrid material was further applied as cathode electrode to prepare lithium battery. And the structure, morphology, electrochemistry and battery performance of the prepared material were studied in detail. FTIR spectra show that hybrid materials have been successfully prepared. SEM results showed that with the increase of p-phenylenediamine monomer in aromatic amine copolymer, the morphology of hybrid materials gradually decreased from the granular morphology of HCF/PANI to a relatively flat stacking state. Cyclic voltammetric (CV) test showed that the redox reaction for arylamine polymer and HCF presented two obvious characteristic peaks. And the introduction of a small amount of p-phenylenediamine monomer caused the oxidation/reduction potential of hybrid material (HCF/P(ANI:pPD=5:1)) to be significantly close each other and the peak space to decrease. With further increasing the proportion of p-phenylenediamine monomer in aromatic amine copolymer, the oxidation peak and reduction peak of hybrid material move reversely to high potential and low potential respectively. Battery performance studies showed that the initial charge-discharge specific capacities of HCF/PANI, HCF/P(ANI:pPD=5:1), HCF/P(ANI:pPD=3:1), HCF/P(ANI:pPD=1:1), HCF/PpPD were 191.3 and 103.5 $\mathrm{mAh} \cdot \mathrm{g}^{-1}, 105.8$ and $82.8 \mathrm{mAh} \cdot \mathrm{g}^{-1}, 86.9$ and $73.9 \mathrm{mAh} \cdot \mathrm{g}^{-1}, 55.4$ and $28.1 \mathrm{mAh} \cdot \mathrm{g}^{-1}, 19.2$ and $12.6 \mathrm{mAh} \cdot \mathrm{g}^{-1}$, respectively With the increase of p-phenylenediamine comonomers in hybrid materials, the cyclic stability of electrode materials has been improved to some extent. At the same time, the hybrid material has good rate retention.
\end{abstract}

Keywords: Prussian Blue, Polyphenyldiamine, Lithium Battery, Electrochemical Properties

\section{铁氧酸掺杂芳胺共聚物杂化材料的制备及其电化学性能规律研究}

徐立环", 马娇娇, 孙越, 郭鹏举, 韩冰, 王国胜, 苏畅

化学工程学院, 沈阳化工大学, 沈阳, 中国

邮箱:

xulihuanss@163.com(徐立环), suchang@syuct.edu.cn（苏畅）

摘要: 以制备的铁㲵酸 ( $\mathrm{HCF}$ ) 为具有电化学活性的掺杂酸, 采用原位合成法制备了 $\mathrm{HCF} / \mathrm{PANI}, \mathrm{HCF} / \mathrm{P}(\mathrm{ANI}: \mathrm{pPD}=5: 1)$, $\mathrm{HCF} / \mathrm{P}(\mathrm{ANI}: \mathrm{pPD}=3: 1), \mathrm{HCF} / \mathrm{P}(\mathrm{ANI}: \mathrm{pPD}=1: 1), \mathrm{HCF} / \mathrm{PpPD}$ 五种杂化共聚物。并以制备的杂化材料为正极制备锂电池, 研究制备材料的结构、形貌、电化学和电池性能变化规律。FTIR光谱表明杂化材料已被成功制备。SEM结果表明, 随 着芳胺共聚物中对苯二胺单体的增加, 杂化材料的形貌由HCF/PANI的颗粒状形貌逐渐变细, 并形成较为平整的堆积 态。循环伏安测试表明, 芳胺聚合物和铁㲵化物的氧化还原反应具有明显特征峰, 少量对苯二胺单体的引入使杂化材 料（HCF/P(ANI:pPD=5:1)）的氧化/还原电位两对氧化/还原峰的明显靠近且峰间距的降低。随着进一步增加芳胺共聚 
物中对苯二胺单体的比例, 杂化材料的氧化峰值与还原峰值又分别向高电位和低电位移动。电池性能研究表明, $\mathrm{HCF} / \mathrm{PANI}, \mathrm{HCF} / \mathrm{P}(\mathrm{ANI}: \mathrm{pPD}=5: 1)$, HCF/P(ANI:pPD=3:1), HCF/P(ANI:pPD=1:1)和(e)HCF/PpPD的首次充放电比容量 分别为 191.3 和 $103.5 \mathrm{mAh} \cdot \mathrm{g}^{-1} 、 105.8$ 和 $82.8 \mathrm{mAh} \cdot \mathrm{g}^{-1} 、 86.9$ 和 $73.9 \mathrm{mAh} \cdot \mathrm{g}^{-1} 、 55.4$ 和 $28.1 \mathrm{mAh} \cdot \mathrm{g}^{-1} 、 19.2$ 和 $12.6 \mathrm{mAh} \cdot \mathrm{g}^{-1}$ 。随着 杂化材料中对苯二胺共聚单体的增加, 电极材料的循环稳定性得到一定改善和提高。同时杂化材料具有较好的倍率保 持率。

关键词: 聚苯二胺, 普鲁士蓝, 锂电池, 电化学性能

\section{1. 引言}

近年来, 在材料科学领域中, 新型有机/无机杂化材 料正引起人们的广泛关注。该类材料由于有机和无机组分 的协同效应, 使其具有突出的性能, 在光、电、磁等领域 具有广泛的应用前景 $[1-3]$ 。

有机共轭聚合物(COPs) 是一类非常特殊的聚合物功 能材料, 由于其独特的共轭结构, 导电性, 电化学活性, 使它们成为功能聚合物功能材料研究的热点 [4-9]。比如, 芳胺聚合物中的聚苯胺在常温下经过化学和电化学掺杂 过程使其具有优异的导电性能，并且由于其易于合成、原 料易得、结构多样化、掺杂原理独特、导电性和稳定性好 等优势, 是目前最具应用前景的导电聚合物。而聚苯二胺 作为一种重要的芳香二胺类聚合物, 因为其大分子结构中 含有比聚苯胺较多的活性自由氨基和亚胺基, 并且还拥有 多功能性, 近年来已被广泛关注[10-15]。

普鲁士蓝(prussian blue, PB)及其衍生物是一种混合价 态的化合物。其具有优良的电化学可逆性, 高度的稳定性, 容易制备等优点, 因此在化学修饰电极、电显示、电催化、 二次储能电池等方面有很大的应用前景[15-29]。

本论文通过化学方法制备具有电化学活性的铁氰酸化 合物, 并以制备的上述铁氧酸为掺杂剂和电化学活性物质, 采用原位聚合法制备不同苯胺和对苯二胺单体比例的的系 列铁氰酸掺杂苯胺-对苯二胺共聚物杂化材料。同时以制备 的系列杂化材料为锂电池正极, 研究不同单体投料比对杂 化电极材料的结构、形貌、电化学和电池性能影响规律。

\section{2. 实验部分}

\section{1. 实验材料与药品}

实验过程中采用的原料有高氯酸 $\left(\mathrm{HClO}_{4}, 70-72 \%\right.$, AR）天津市金源化工有限公司; 铁氭化钾 $\left(\mathrm{K}_{3} \mathrm{Fe}(\mathrm{CN})_{6}\right.$, $99.5 \%, A R$ ）、苯胺（Aniline，99.5\%，AR）、对苯二胺 (CP) 国药集团化学试剂有限公司; Super p li 导电炭黑 (特密高) 、 PVDF、电解液 $\left(1 \mathrm{~mol} / \mathrm{L} \mathrm{LiPF}_{6} \mathrm{EC}: \mathrm{DMC}\right.$ : $\mathrm{EMC}=1: 1: 1(\mathrm{w} / \mathrm{w}))$ 均购自太原迎泽区力之源电池销 售部。

\section{2. 铁氬酸及其掺杂芳胺共聚物杂化材料制备}

铁氰酸制备: 先用量筒量取 $100 \mathrm{~mL}$ 去离子水倒入 $250 \mathrm{~mL}$ 的烧杯中，再用规格为 $10 \mathrm{~mL}$ 的量筒量取 $17.8 \mathrm{~mL}$ 的 高氯酸溶液, 缓慢倒入烧杯中, 配制成浓度为 $2.1 \mathrm{~mol} / \mathrm{L}$ 的
$100 \mathrm{~mL}$ 高氯酸溶液; 称量 $5.679 \mathrm{~g}$ 铁氧化钾, 溶解到 $25 \mathrm{~mL}$ 去 离子水中; 然后量取 $25 \mathrm{~mL}$ 上述配制好的高氯酸溶液加入 到铁氰化钾溶液中; 经过均匀搅拌用布氏漏斗过滤掉白色 沉淀物高氯酸钾, 得到黄色溶液铁氰酸。

铁氰酸掺杂苯胺-对苯二胺共聚物制备: 配制成 $0.2 \mathrm{~mol} / \mathrm{L}$ 的苯胺溶液; 量取 $25 \mathrm{~mL}$ 配制好的苯胺溶液倒入 $250 \mathrm{~mL}$ 的烧杯中, 用胶头滴管取上述得到的黄色溶液铁氰 酸逐滴滴加到苯胺溶液中; 在室温下搅拌反应 3 天, 然后 过滤、水洗 3 次, 最后得到墨绿色滤饼进行干燥至少 $12 \mathrm{~h}$ 以上。最终产物为黑绿色粉末。

用天平称量 $2.163 \mathrm{~g}$ 对苯二胺, 溶解到去离子水中, 配 制成 $0.2 \mathrm{~mol} / \mathrm{L}$ 的 $100 \mathrm{~mL}$ 溶液; 再用量筒量取 $25 \mathrm{~mL}$ 配制好的 对苯二胺溶液, 取上述得到的黄色溶液铁氰酸逐滴滴加到 对苯二胺溶液中; 放入转子用磁力加热搅拌器在室温下摚 拌 3 天, 然后取下溶液进行 2 遍以上的水洗、过滤，最后得 到黑色滤饼进行干燥至少 $12 \mathrm{~h}$ 以上。最终产物为黑色粉末 的产品 (HCF/PANI)。

铁氰酸掺杂苯胺-对苯二胺共聚物的制备具体如下, 分别配制苯胺+对苯二胺浓度为 $0.2 \mathrm{~mol} / \mathrm{L}$ 的单体溶液 $25 \mathrm{~mL}$, 将制备铁氰酸逐滴滴加到不同摩尔比的混合溶液中; 具体 反应过程如HCF/PANI制备过程。其中混合单体溶液中苯 胺:对苯二胺摩尔比分别为 $5: 1,3: 1,1: 1,0: 1$, 所得对应 产物 分 别表达为 $\mathrm{HCF} / \mathrm{P}(\mathrm{ANI}: \mathrm{pPD}=5: 1)$, $\mathrm{HCF} / \mathrm{P}(\mathrm{ANI}: \mathrm{pPD}=3: 1)$, HCF/P(ANI:pPD=1:1)和HCF/PpPD。

\section{3. 表征与测试}

红外光谱 (FTIR) 采用美国热电公司NEXUS470型傅 立叶变换红外光谱仪采集，测试波段为 $4000-400 \mathrm{~cm}^{-1}$; 扫 描电镜分析（SEM）采用日本电子公司JSM-6360LV型场 发射扫描电镜观察样品的表面形貌、颗粒大小和颗粒的分 布情况; 循环伏安测试采用上海华辰CHI660E型电化学工 作站对半电池进行循环伏安测试, 扫描的速度设置为 $1 \mathrm{mV} / \mathrm{s}$, 电压范围设置为 $2.5-4.2 \mathrm{~V}$ 。测试过程中, 以金属锂 片作为对电极和参比电极; 材料的电池性能测试在武汉金 诺电子有限公司的 LAND电池测试系统 (CT2001A, $5 \mathrm{~V} / 10 \mathrm{~mA}$ ) 上进行充放电测试, 电压范围为2.5-4.2V, 以 $50 \mathrm{~mA} \cdot \mathrm{g}^{-1}$ 的充放电速率进行恒流充放电性能测试。采用恒 电流充放电法测试电池的比容量和循环性能, 并且在50、 100、200、500 $\mathrm{mA} \cdot \mathrm{g}^{-1}$ 的充放电速率进行阶梯充放电性能 测试。交流阻抗测试使用上海辰华CHI660E型电化学工作 站对样品进行交流阻抗测试, 频率范围设置为 $0.01 \mathrm{~Hz}-100 \mathrm{kHz}$, 振幅 $5 \mathrm{mV}$ 。阻抗测试采用两电极体系, 分别以活性物质为工作电极, 电池的负极 (锂片) 为对电 
极和参比电极, 在放电后的平台电压下对实验半电池进行 测试。

\section{4. 电极制备及半电池组装}

电极的制备: 采用涂膜法制备电极, N-甲基吡咯烷 酮(NMP)作为溶剂, 将原料按照质量比 $\mathrm{m}$ (杂化材料) : $\mathrm{m}$ (导电剂乙炔黑) : $\mathrm{m}(\mathrm{PVDF})=50: 40: 10$ 的比例混合 成正极浆液, 再将浆液涂在预处理过的铝䇴上, 在真空 干燥箱中于 $70^{\circ} \mathrm{C}$ 下干燥 $24 \mathrm{~h}$, 压片 $(16 \mathrm{Mpa})$ 后得到正 极片。

半电池的组装: 半电池组装是在高纯 $\operatorname{Ar}$ 保护的手套 箱中完成, 手套箱中的湿度和氧含量保持在 $1 \mathrm{ppm}$ 以下。 以金属锂片为对电极和参比电极, $1 \mathrm{~mol} / \mathrm{LLiPF}_{6} \mathrm{EC}$ : $\mathrm{DMC}: \mathrm{EMC}=1: 1: 1(\mathrm{w} / \mathrm{w})$ 溶液为电解液, 聚丙烯 微孔膜(Celgard2300)为隔膜, 组装成CR2032扣式半电池。 组装好的电池进行封口并静置 $12 \mathrm{~h}$, 以便电解液能够在 电池两极之间扩散均匀, 使电解液与电池的两极完全接 触。

\section{3. 结果与讨论}

\subsection{FTIR分析}

图 1 为样品 $\mathrm{HCF} / \mathrm{PANI}, \mathrm{HCF} / \mathrm{P}(\mathrm{ANI}: \mathrm{pPD}=5: 1)$, $\mathrm{HCF} / \mathrm{P}(\mathrm{ANI}: \mathrm{pPD}=3: 1), \mathrm{HCF} / \mathrm{P}(\mathrm{ANI} / \mathrm{pPD}=1: 1)$ 和 $\mathrm{HCF} / \mathrm{PpPD}$ 的红外光谱。如图1所示, 五种杂化材料在 2060-2080 $\mathrm{cm}^{-1}$ 附近存在-CN伸缩振动特征吸收峰, 证明 $\mathrm{HCF}$ 已经掺杂进 了聚合物中。同时, 对比HCF/PANI和HCF/PpPD杂化材料 特征吸收峰, HCF/P(ANI:pPD=5:1), HCF/P(ANI:pPD=3:1) 和 HCF/P(ANI:pPD $=1: 1$ ) 三种芳胺共聚物杂化材料在 $1575 \mathrm{~cm}^{-1}$ 和 $1510 \mathrm{~cm}^{-1}$ 处特征吸收峰归属于醌式结构的伸缩
振动特征吸收峰和苯环伸缩振动特征吸收峰; 在 $1289 \mathrm{~cm}^{-1}$ 特征吸收峰处归属于- $\mathrm{HN}-\mathrm{C}_{6} \mathrm{H}_{4}-\mathrm{NH}-$ 中的 C-N 伸缩振动特 征吸收峰; 在 $1175 \mathrm{~cm}^{-1}$ 处特征吸收峰处归属于醌式结构的 $\mathrm{C}-\mathrm{N}$ 伸缩振动; 在 $828 \mathrm{~cm}^{-1}$ 处特征吸收峰处归属于 1,4 取代 苯环上的 $\mathrm{C}-\mathrm{H}$ 键平面外剪切振动特征吸收峰; 在 $515 \mathrm{~cm}^{-1}$ 处特征吸收峰处归属于芳环弯曲振动特征吸收峰。上述特 征说明三种不同比例的苯胺-对苯二胺共聚物是以头尾连 接形式聚合的, 聚合物中包括苯胺和对苯二胺两种功能单 体, 同时, 采用原位掺杂聚合法成功将铁氰酸分子掺杂进 入聚芳胺共聚物材料体系。

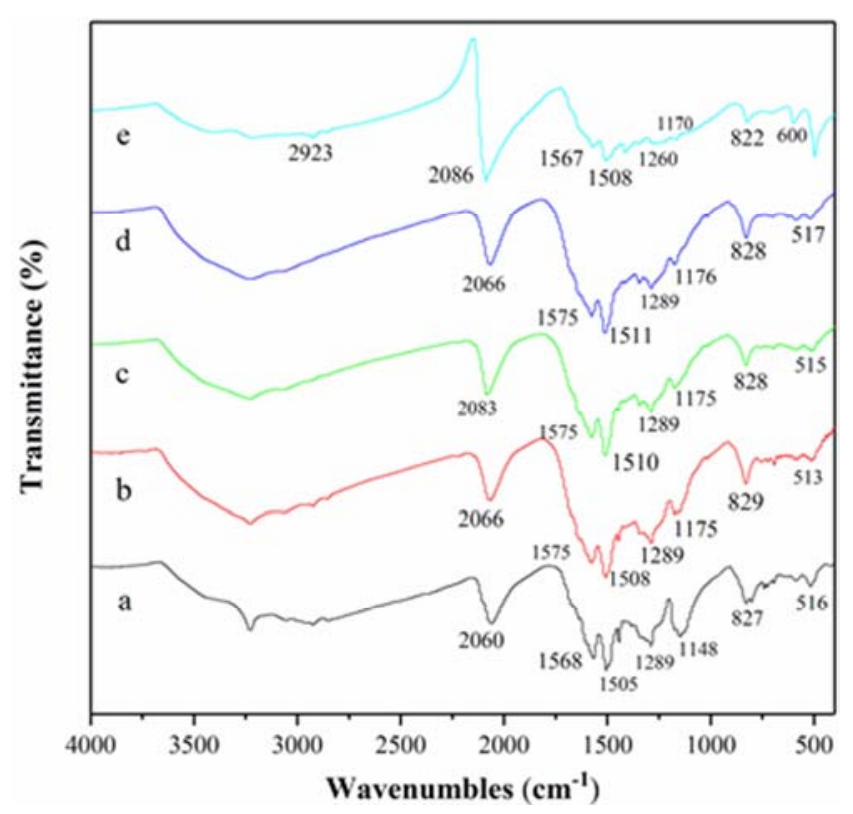

图1 (a)HCF/PANI, (b)HCF/P(ANI:pPD=5:1), (c)HCF/P(ANI:pPD=3:1), (d)HCF/P(ANI:pPD=1:1), (e)HCF/PpPD的红外光谱图。

\subsection{SEM分析}
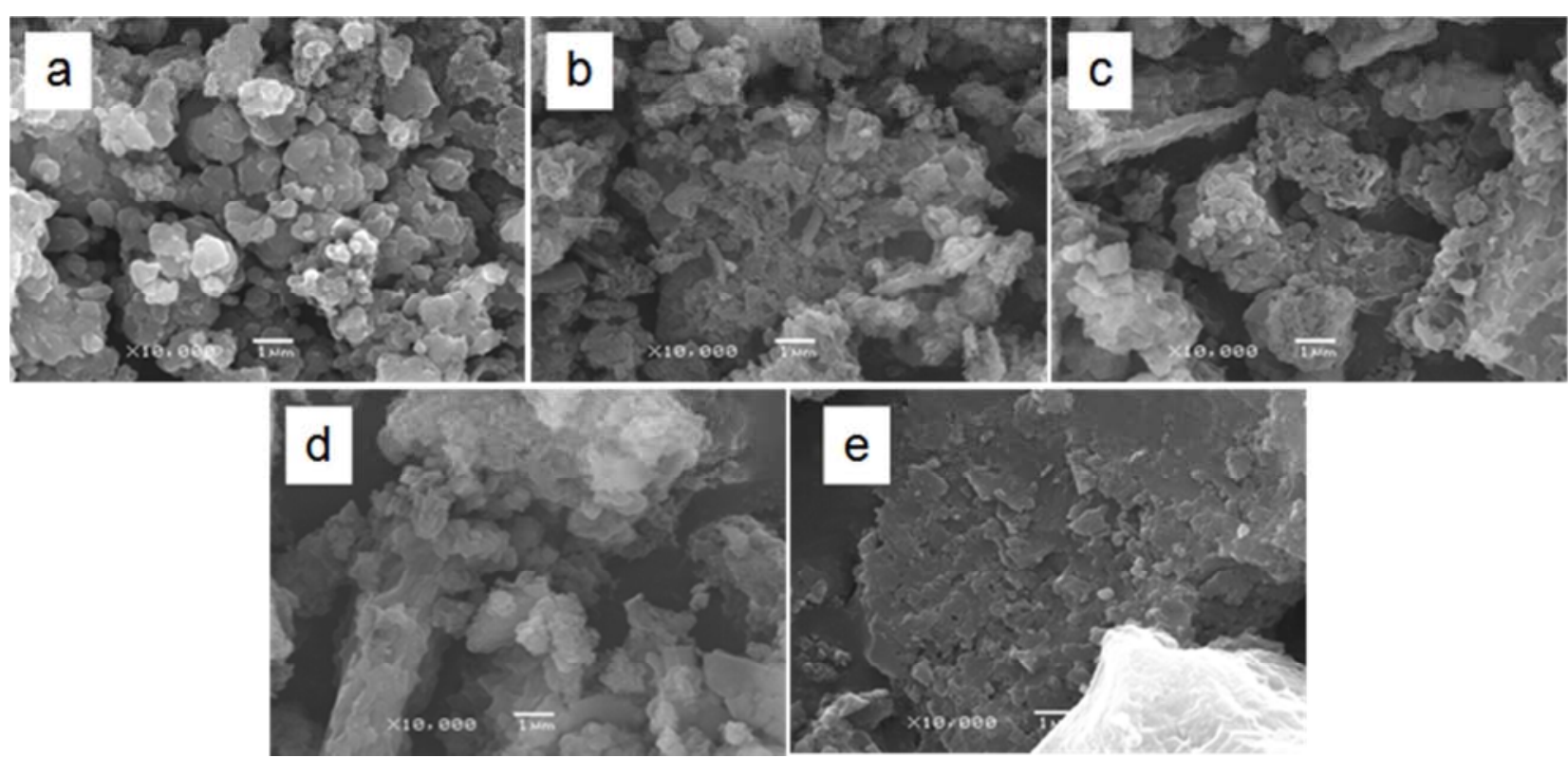

图2 (a)HCF/PANI，(b)HCF/P(ANI:pPD=5:1)，(c)HCF/P(ANI:pPD=3:1)，(d)HCF/P(ANI:pPD=1:1)，(e)HCF/PpPD的SEM图。 


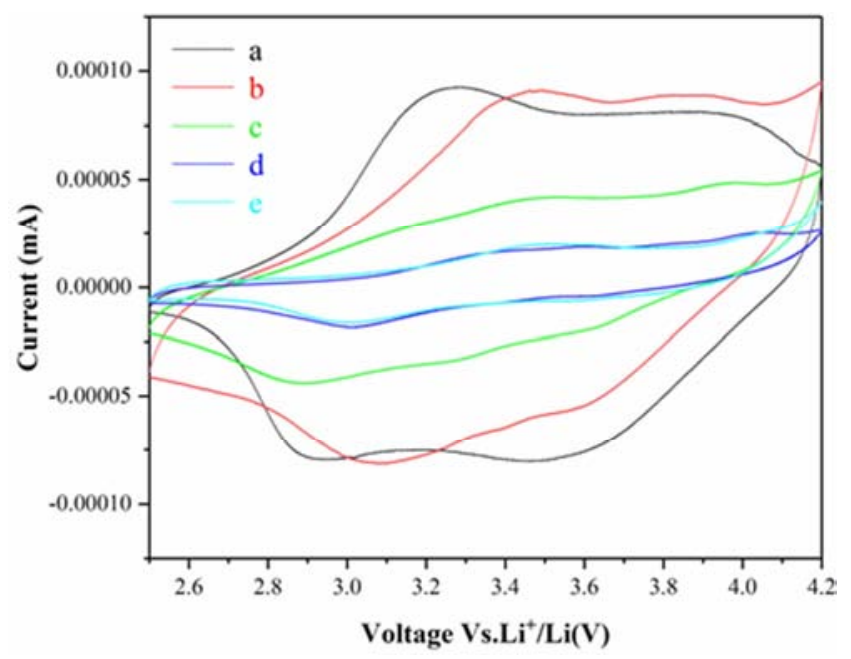

图3 (a)HCF/PANI, (b)HCF/P(ANI:pPD=5:1), (c)HCF/P(ANI:pPD=3:1), (d)HCF/P(ANI:pPD=1:1), (e)HCF/PpPD的循环伏安曲线图。

图 2 分别为 HCF/PANI, HCF/P(ANI:pPD=5:1), HCF/P(ANI:pPD=3:1), HCF/P(ANI:pPD=1:1)和HCF/PpPD五 种杂化材料的SEM图。由图可以看出, HCF/PANI的杂化材 料的表现为较大的颗粒状形貌, 而随着聚对苯二胺单体的引 入, 聚芳胺共聚物杂化材料体系的颗粒变小。当芳胺聚合物 完全为PpPD纯材料时, HCF/PpPD杂化材料的颗粒甚至变得 更小, 以至于材料表面变得较为平滑, 呈现密集堆积平面形 貌。研究结果表明, 芳胺共聚物的组成对铁氧酸掺杂的芳胺 共聚物杂化材料的形貌产生明显影响, 这种差异可能是由于 单体阳离子自由基结构的稳定性的差异所引起分子组成和 聚合力变化, 并最终导致材料的二次堆积形貌的变化。

\section{3. 循环伏安测试分析}

图3为五种杂化材料的循环伏安曲线，扫描速率均为 $1 \mathrm{mV} / \mathrm{s}$ 。从图中可以看出, $\mathrm{HCF} / \mathrm{PANI}$ 杂化材料分别在 3.24/2.89V和3.94/3.53V位置出现两对明显的氧化还原峰, 分 别对应 $\mathrm{Fe}(\mathrm{CN})_{6}^{3-1 / 4}$ 和聚苯胺氧化/还原反应峰, 其峰间距为 $0.35 \mathrm{~V}$ 和 $0.41 \mathrm{~V}$ 。相对应地, 随着对苯二胺的引入, HCF/P(ANI:pPD=5:1)杂化材料的氧化/还原峰位置分别出现 在3.46/3.12V和3.89/3.61V位置, 其峰间距为 $0.34 \mathrm{~V}$ 和 $0.27 \mathrm{~V}$ 。 其两对氧化/还原峰的明显靠近且峰间距的降低表明, 少量 对苯二胺芳香单体的引入能明显改善杂化材料的极性并降 低复合电极的极化。随着进一步增加芳胺共聚物中对苯二胺 单体的比例, $\mathrm{HCF} / \mathrm{P}(\mathrm{ANI}: \mathrm{pPD}=3: 1) 、 \mathrm{HCF} / \mathrm{P}(\mathrm{ANI}: \mathrm{pPD}=1: 1)$ 、 $\mathrm{HCF} / \mathrm{pPD}$ 三种杂化材料的氧化峰值与还原峰值又分别向高 电位和低电位移动, 表明进一步增加对苯二胺单体的比例降 低了芳胺共聚物材料的共轭, 使杂化电极材料的极化增加, 导致氧化峰和还原峰的距离增大。同时, 氧化还原峰面积随 着对苯二胺单体的增加逐渐降低, 表明其电存储性能变差。

\section{4. 充放电性能测试}

图 4 为 $\mathrm{HCF} / \mathrm{PANI}, \mathrm{HCF} / \mathrm{P}(\mathrm{ANI}: \mathrm{pPD}=5: 1)$, HCF/P(ANI:pPD=3:1), HCF/P(ANI:pPD=1:1), HCF/PpPD五 种杂化材料在 $1 \mathrm{~mol} / \mathrm{LLiPF}_{6} \mathrm{EC}: \mathrm{DMC}: \mathrm{EMC}=1: 1: 1(\mathrm{w} / \mathrm{w})$
电解质中, 以 $50 \mathrm{~mA} \cdot \mathrm{g}^{-1}$ 恒定充放电速率下测试的首次充放电 曲线。如图所示, $\mathrm{HCF} / \mathrm{PANI}, \mathrm{HCF} / \mathrm{P}(\mathrm{ANI}: \mathrm{pPD}=5: 1)$, HCF/P(ANI:pPD=3:1), HCF/P(ANI:pPD=1:1)和(e)HCF/PpPD 的首次充放电比容量分别为 191.3 和 $103.5 \mathrm{mAh} \cdot \mathrm{g}^{-1} 、 105.8$ 和 $82.8 \mathrm{mAh} \cdot \mathrm{g}^{-1} 、 86.9$ 和 $73.9 \mathrm{mAh} \cdot \mathrm{g}^{-1} 、 55.4$ 和 $28.1 \mathrm{mAh} \cdot \mathrm{g}^{-1} 、 19.2$ 和 $12.6 \mathrm{mAh} \cdot \mathrm{g}^{-1}$ 。并且, HCF/PANI杂化电极材料呈现一个放 电平台, 这是由于铁氰酸掺杂所致。随着芳胺共聚物中对苯 二胺单体的引入，充放电比容量逐渐降低，这与CV测试过 程中逐渐减小的曲线面积相一致。

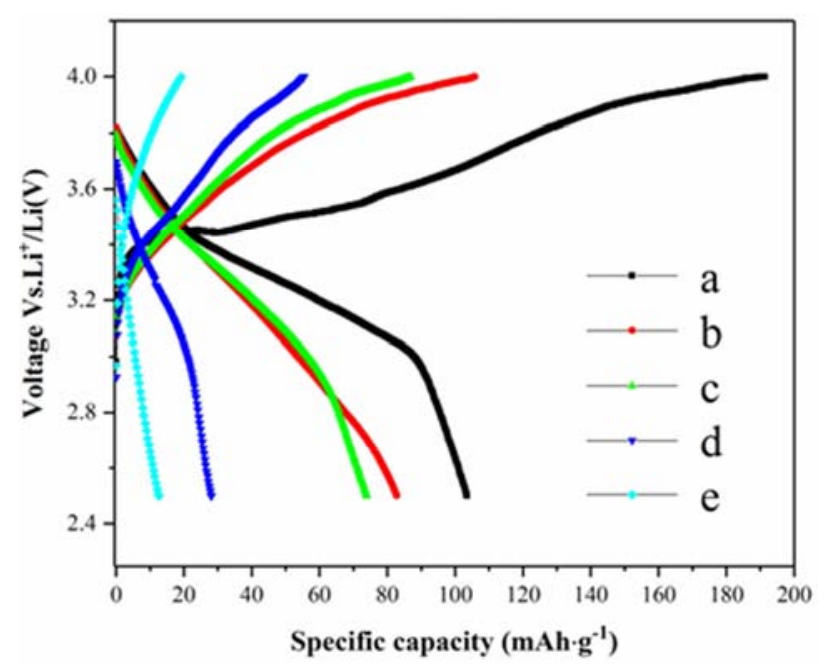

图4 (a)HCF/PANI, (b)HCF/P(ANI:pPD=5:1), (c)HCF/P(ANI:pPD=3:1), (d)HCF/P(ANI:pPD=1:1), (e) HCF/PpPD在 $50 \mathrm{~mA}^{-1} \mathrm{~g}^{-1}$ 充放电速率下的首次 充放电曲线。

该杂化材料的储能机制可解释为: 铁氧酸掺杂聚苯胺、 聚对苯二胺以及苯胺和对苯二胺的共聚物得到的杂化材 料中的阴离子针定在杂化物上, 阳离子由有机溶剂电解质 中的 $\mathrm{Li}^{+}$提供, 分别在在还原和氧化时嵌入和脱嵌。而相 比于传统小分子无机酸掺杂导电有机物过程中, 伴随着阴 离子的嵌入/脱嵌过程所发生氧化/还原反应。

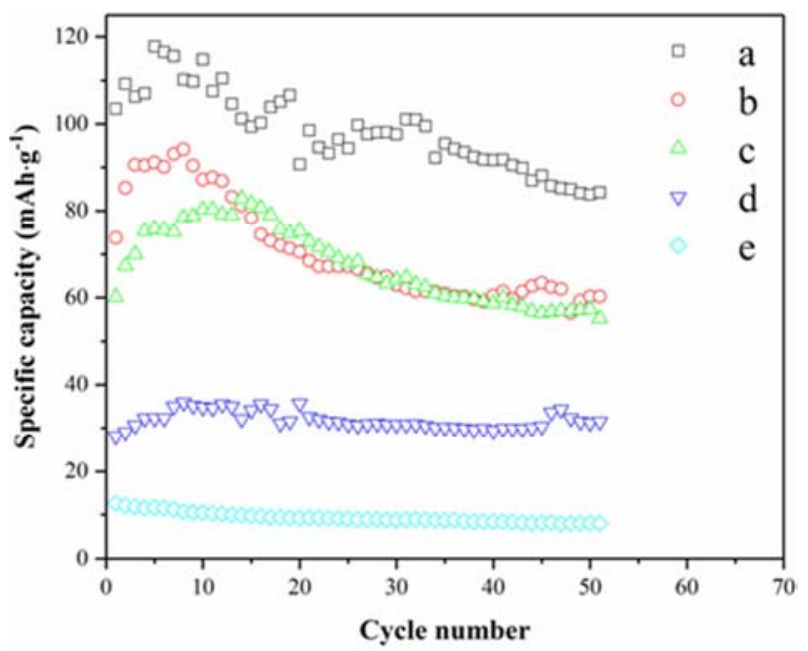

图5 (a)HCF/PANI, (b)HCF/P(ANI:pPD=5:1), (c)HCF/P(ANI:pPD=3:1), (d)HCF/P(ANI:pPD=1:1), (e) HCF/PpPD在50mA. $\mathrm{g}^{-1}$ 速率下放电的循环性 能图。 
图5为五种杂化材料电池的充放电循环性能测试图。 如图所示, HCF/PANI, HCF/P(ANI:pPD=5:1), HCF/P(ANI:pPD=3:1), HCF/P(ANI:pPD=1:1), HCF/PpPD 五种杂化材料在循环 10 圈以内比容量都有缓慢上升, 然后 又逐渐降低, 起始的容量升高可能是由于电极材料的缓慢 活化过程所致。 $\mathrm{HCF} / \mathrm{PANI}$ 在充放电 50 次后, 放电比容量 从首次的 $103.5 \mathrm{mAh} \cdot \mathrm{g}^{-1}$ 先上升一段, 循环 10 圈左右之后缓 慢降至 $84.3 \mathrm{mAh} \cdot \mathrm{g}^{-1}$, 容量损失率仅为 $18.8 \%$ 。而 HCF/P(ANI:pPD=5:1) 和HCF/P(ANI:pPD=3:1)也展示出了 相似的特性, 在 50 次充放电过后, 其放电比容量分别从首 次的 $73.8 \mathrm{mAh} \cdot \mathrm{g}^{-1} 、 60.2 \mathrm{mAh} \cdot \mathrm{g}^{-1}$ 先上升一段, 循环 10 圈左 右之后慢慢降至 $60.3 \mathrm{mAh} \cdot \mathrm{g}^{-1}$ 和 $55.2 \mathrm{mAh} \cdot \mathrm{g}^{-1}$, 容量损失率 分别仅为 $18.3 \%$ 和 $8.3 \%$ 。然而, HCF/P(ANI: $\mathrm{pPD}=1: 1)$ 和 HCF/PpPD放电容量具有较高的保持率。相对而言, 研究 发现, 随着杂化材料中对苯二胺共聚单体的增加, 电极材 料的循环稳定性得到一定改善和提高, 这可能是由于形成 的芳胺共聚中增加的氨基基团提高了与铁氰酸根离子的 静电相互作用, 提高了电极材料的循环稳定性。

图 6 是 HCF/PANI , HCF/P(ANI:pPD=5:1) , HCF/P(ANI:pPD=3:1), HCF/P(ANI:pPD=1:1), HCF/PpPD 五种杂化材料在 $50 、 100 、 200 、 500 \mathrm{~mA} \cdot \mathrm{g}^{-1}$ 充放电速率下的 充放电曲线。从图 5 中可以看出, 五种杂化材料在 50-500 $\mathrm{mA} \cdot \mathrm{g}^{-1}$ 充放电速率下, 各阶梯循环中的首次放电比容 量分别为 $105 、 70.8 、 60.2 、 22$ 和 $12.5 \mathrm{mAh} \cdot \mathrm{g}^{-1}$, 到 $200 \mathrm{~mA} \cdot \mathrm{g}^{-1}$
放电结束, 五种杂化材料的比容量为89.90、65.49、66、33 和 $10.1 \mathrm{mAh} \cdot \mathrm{g}^{-1}$ 。表明材料具有相对较好的倍率保持性。另 外, 如图7所示, 从不同放电倍率曲线特征可以看出, 五钟 杂化电极材料的放电曲线在不同的放电倍率下具有相似的 放电曲线特征, 表明, 杂化电极材料在不同的放电倍率条 件下具有稳定的物质结构, 并未发生物质结构的明显变化。

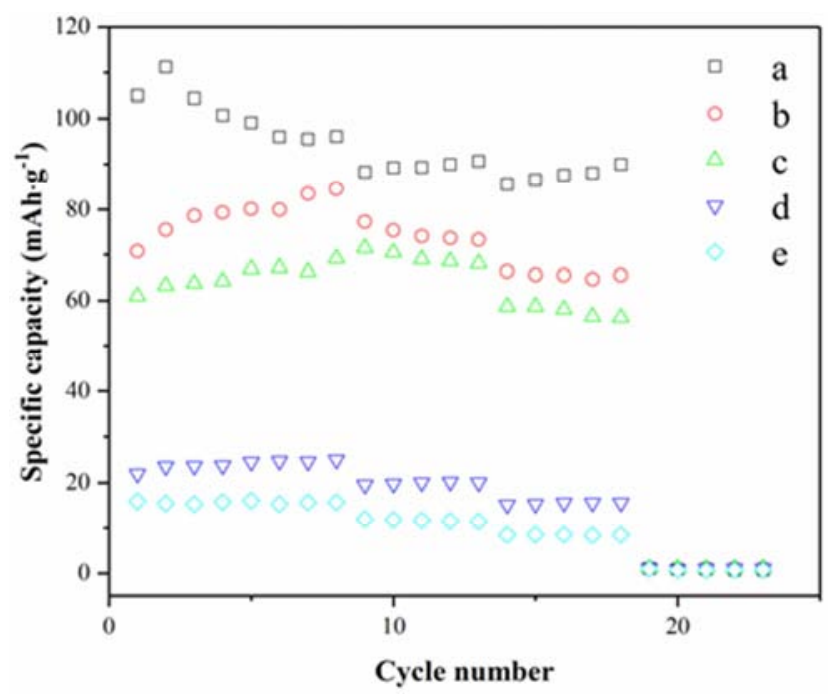

图6 (a)HCF/PANI, (b)HCF/P(ANI:pPD=5:1), (c)HCF/P(ANI:pPD=3:1), (d)HCF/P(ANI:pPD=1:1), (e)HCF/PpPD在50, 100, 200, 500mA-g-1四 种不同放电倍率下的循环性能图。

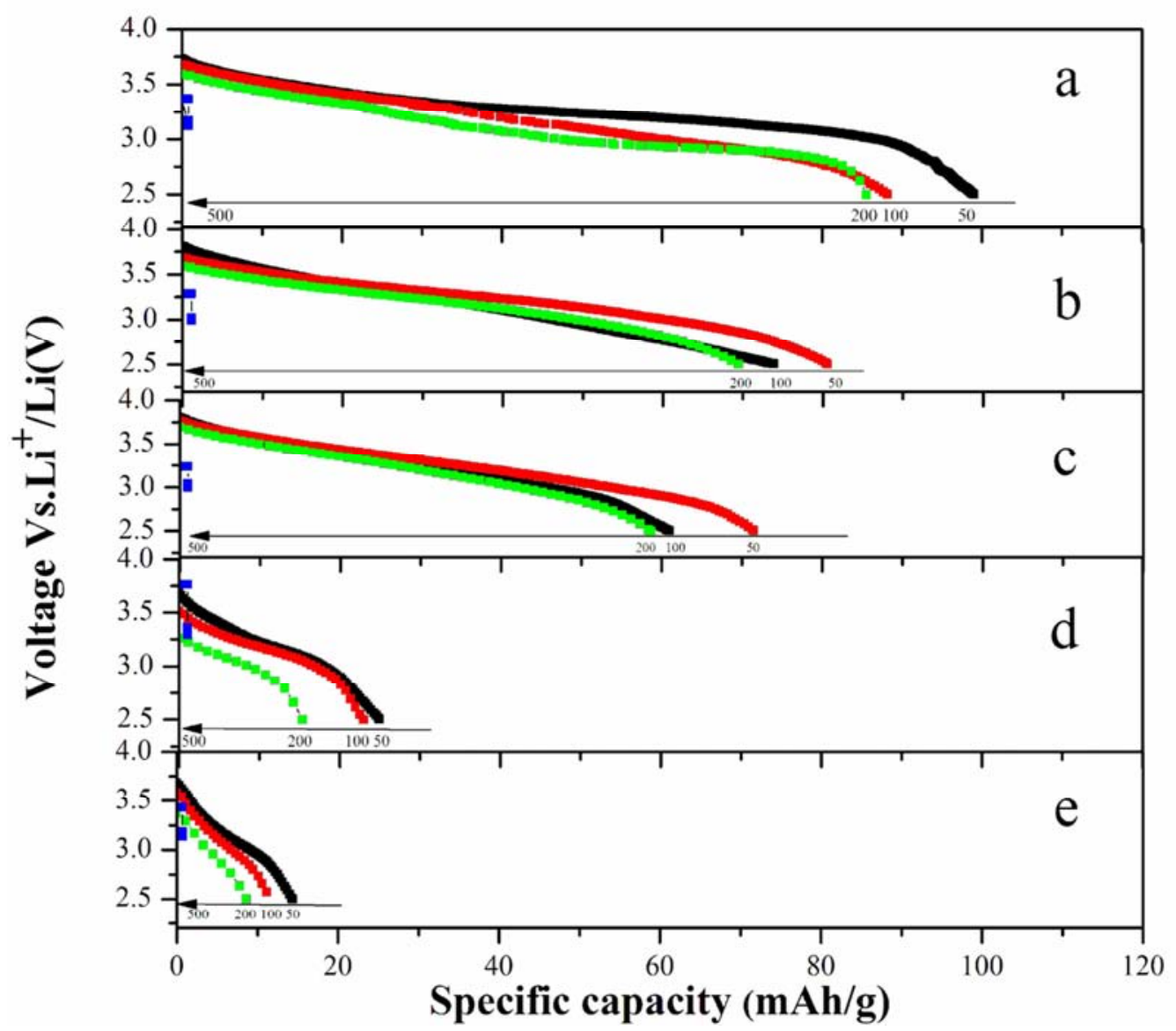

图7 (a)HCF/PANI, (b)HCF/P(ANI:pPD=5:1)，(c)HCF/P(ANI:pPD=3:1)，(d)HCF/P(ANI:pPD=1:1)，(e)HCF/PpPD在50，100，200，500mA.g-1 四种不同 放电速率下的放电曲线图。 


\section{5. 交流阻抗测试}

图8给出了五种杂化材料的交流阻抗谱。由图可见， 样品的交流阻抗图谱由半圆形的高频区和直线形的低频 区组成。图谱的起始位置在高频区与 $\mathrm{Z}_{\mathrm{re}}$ 轴上的交点对应于 电解液的欧姆电阻 $\left(\mathrm{R}_{\mathrm{e}}\right)$, 半圆的前半部分是 $\mathrm{Li}^{+}$通过电极和 电解液的SEI膜的阻抗, 半圆的后半部分则是电荷的转移 电阻 $\left(\mathrm{R}_{\mathrm{ct}}\right)$ 。而低频区的直线部分体现的是 $\mathrm{Li}^{+}$在材料内部的 扩散, 也叫Warburg扩散阻抗 $\left(Z_{\mathrm{w}}\right)$ 。因此, 图中半圆弧的 直径大小说明了电极界面处的电荷转移的电阻大小, 电极 的圆弧越大意味着 $\mathrm{Li}^{+}$在电极上嵌入/脱出时所对应的电荷 转移过程的阻力越大, 反之, 则越小。由图中可知, 曲线 的形状相似, 并且高频区的半圆形很小，这说明虽然正极 材料不同, 但是其对于电解质溶液中离子的电导率和迁移 率的影响是非常小的。然而值得注意的是，从图中可以发 现不同曲线的半圆弧直径相差较大, 说明在这五种材料制 备的扣式电池中, 电荷转移阻抗 $\left(\mathrm{R}_{\mathrm{ct}}\right)$ 部分存在着很大的区 别。五种杂化材料电荷转移阻抗依次分别约为 $2150 \Omega$ 、 $2520 \Omega 、 2910 \Omega 、 4460 \Omega 、 5620 \Omega$ 。所以可以得到随着杂化 材料中芳胺共聚物中对苯二胺含量的增加, 杂化电极材料 中 $\mathrm{Li}^{+}$嵌入/迁出所对应的电荷转移过程的阻力逐渐变小。

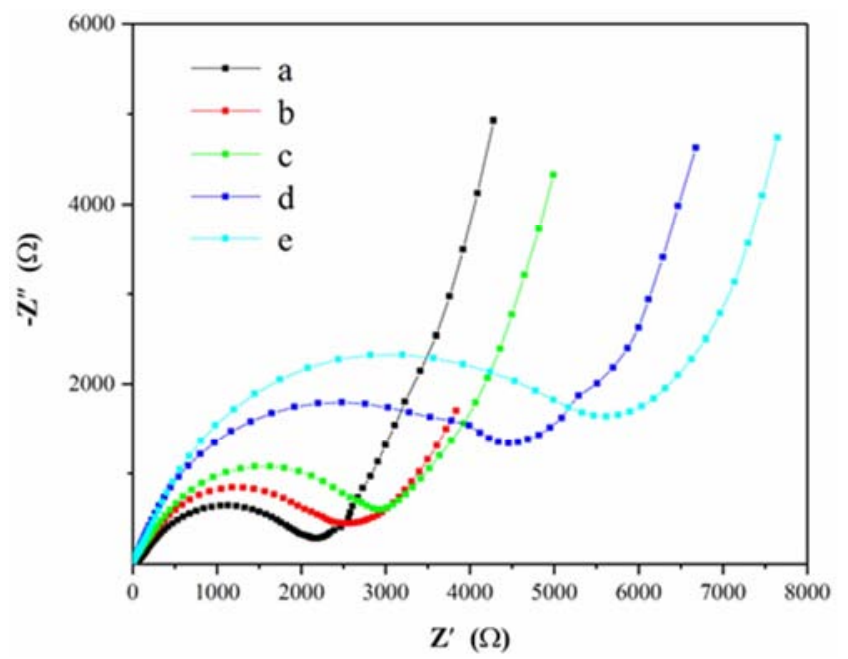

图8 (a)HCF/PANI, (b)HCF/P(ANI:pPD=5:1), (c)HCF/P(ANI:pPD=3:1), (d)HCF/P(ANI:pPD=1:1), (e)HCF/PpPD的交流阻抗图。

\section{4. 结论}

本文首先采用原位合成法制备了 $\mathrm{HCF} / \mathrm{PANI}$, $\mathrm{HCF} / \mathrm{P}(\mathrm{ANI}: \mathrm{pPD}=5: 1) \quad, \quad \mathrm{HCF} / \mathrm{P}(\mathrm{ANI}: \mathrm{pPD}=3: 1) \quad$ ， $\mathrm{HCF} / \mathrm{P}(\mathrm{ANI}: \mathrm{pPD}=1: 1)$, HCF/PpPD五种杂化共聚物。FTIR 光谱测试证实铁氭酸掺杂聚芳胺共聚物杂化材料已被成 功制备。SEM测试结果表明, 芳胺共聚物的组成对铁氰酸 掺杂的芳胺共聚物杂化材料的形貌产生明显影响。随着芳 胺共聚物中对苯二胺单体的增加, 杂化材料的形貌由 $\mathrm{HCF} / \mathrm{PANI}$ 的颗粒状形貌逐渐变细, 并形成较为平整的堆 积态。循环伏安测试显示芳胺聚合物的氧化还原过程和铁 氧化物的氧化还原反应具有明显特征峰, 少量对苯二胺单 体的引入使杂化材料 $(\mathrm{HCF} / \mathrm{P}(\mathrm{ANI}: \mathrm{pPD}=5: 1))$ 的氧化/还
原电位两对氧化/还原峰的明显靠近且峰间距的降低。随 着进一步增加芳胺共聚物中对苯二胺单体的比例, $\mathrm{HCF} / \mathrm{P}(\mathrm{ANI}: \mathrm{pPD}=3: 1)$ HCF/P(ANI:pPD=1:1)、HCF/pPD 三种杂化材料的氧化峰值与还原峰值又分别向高电位和 低电位移动, 表明进一步增加对苯二胺单体的比例降低了 芳胺共聚物材料的共轭, 使杂化电极材料的极化增加, 导 致氧化峰和还原峰的距离增大。同时, 氧化还原峰面积随 着对苯二胺单体的增加逐渐降低, 表明其电存储性能变差。 电池性能研究表明, HCF/PANI, HCF/P(ANI:pPD=5:1), $\mathrm{HCF} / \mathrm{P}(\mathrm{ANI}: \mathrm{pPD}=3: 1) \quad, \quad \mathrm{HCF} / \mathrm{P}(\mathrm{ANI}: \mathrm{pPD}=1: 1) \quad$ 和 (e)HCF/PpPD 的首次充放电比容量分别为 191.3 和 $103.5 \mathrm{mAh} \cdot \mathrm{g}^{-1} 、 105.8$ 和 $82.8 \mathrm{mAh} \cdot \mathrm{g}^{-1} 、 86.9$ 和 $73.9 \mathrm{mAh} \cdot \mathrm{g}^{-1}$ 、 55.4 和 $28.1 \mathrm{mAh} \cdot \mathrm{g}^{-1} 、 19.2$ 和 $12.6 \mathrm{mAh} \cdot \mathrm{g}^{-1}$ 。随着杂化材料中 对苯二胺共聚单体的增加, 电极材料的循环稳定性得到一 定改善和提高。同时杂化材料具有较好的倍率保持率。

\section{致谢}

本文为辽宁省自然科学基金项目《聚苯胺 $/ \mathrm{Fe}(\mathrm{CN})_{6}{ }^{3-}$ 石墨烯化碳管杂化锂电池正极材料的研究》(201602591)、 《微介孔结构聚三苯胺衍生物有机锂电池正极材料的研 究》(2015020441); 辽宁省教育厅项目《原位自聚合法制 备导电高分子修饰的普鲁士蓝金属配位化合物锂电池正 极材料的研究》(LQ2017010); 国家自然科学基金一般项 目《含均三嗪结构聚三苯胺微介孔有机锂电池正极材料的 制备与电化学性能研究》(51573099); 辽宁省高校重点实 验室项目《基于聚三苯胺衍生物/石墨烯复合材料的有机 自由基聚合物锂电池正极材料的基础研究》(LZ2016005); 辽宁省高等学校创新人才支持计划项目(LR2017034)资助。

\section{参考文献}

[1] P. Gómez-Romero. Hybrid organic-inorganic materials-in search of synergic activity [J]. Adv. Mater. 2001, 13(3): 163-174.

[2] P. Gómez-Romero, G. Torres-Gómez. Molecular batteries: harnessing $\mathrm{Fe}(\mathrm{CN})_{6}{ }^{3-}$ electroactivity in hybrid polyaniline-hexacyanoferrate electrodes [J]. Adv. Mater. 2000, 12 (19): 1454-1456.

[3] P. Judeinstein, C. Sanchez. Hybrid organic-inorganic materials: A land of multi-disciplinarity [J]. J. Mater. Chem. 1996, 6(4): 511-525.

[4] A. Vadivel Murugan, C. S. Gopinath, K. Vijayamohanan, Electrochemical studies of poly (3,4-ethylenedioxythiophene) PEDOT/VS 2 nanocomposite as a cathode material for rechargeable lithium batteries $[\mathrm{J}]$. Electrochem. Commun. 2005, 7: 213-218.

[5] G. Li, P.G. Pickup, Phys. Ion transport in poly(3,4-ethylenedioxythiophene)-poly(styrene-4-sulfonate) composites [J]. Phys. Chem. Chem. Phys. 2000, 2: 1255-1260. 
[6] K. S. Park, S. B. Schougaard, J. B. Goodenough. Conducting-polymer/iron-redox-couple composite cathodes for lithium secondary batteries [J]. Adv. Mater. 2007, 19: 848-851.

[7] Y. Wang, Y. Shi, L. Pan, Y. Ding, Y. Zhao, Y. Li, Y. Shi, G. Yu. Dopant-enabled supramolecular approach for controlled synthesis of nanostructured conductive polymer hydrogels $[\mathrm{J}]$. Nano Lett. 2015, 15: 7736-7741.

[8] K. A. See, S. Hug, K. Schwinghammer, M. A. Lumley, Y. Zheng, J. M. Nolt, G. D. Stucky, F. Wudl, B. V. Lotsch, R. Seshadri. Lithium charge storage mechanisms of cross-linked triazine networks and their porous carbon derivatives [J]. Chem. Mater. 2015, 27: 3821-3829.

[9] Y. Shi, L. Peng, G. H. Yu. Nanostructured conducting polymer hydrogels for energy storage applications. Nanoscale, 2015, 7: 12796-12806

[10] P. Gajendran, S Vijayan, R. Saraawathi. Investigation of oxygen reduction at platinum loaded poly(o-phenylenediamine) electrode in acid medium [J]. J. Electroanal. Chem. 2007, 601(1-2): 132-138.

[11] X. F. Lu, H. Mao, D. M. Chao. Prepatation and characterization of poly(o-phenylenediamine) microrods using ferric chloride as an oxidant [J]. Mater. Lett, 2007, 61(6): 1400-1043.

[12] H. Yadegari, H. Heli, A. Jabbari. Graphene/poly(ortho-phenylenediamine) nanocomposite material for electrochemical supercapacitor [J]. J. Solid State Electrochem. 2013, 17: 2203-2212.

[13] Z. H. Liu, H. H. Zhou, Z. Y. Huang, W. Y. Wang, F. Y. Zeng, Y. F. Kuang. Graphene covalently functionalized with poly(p-phenylenediamine) as high performance electrode material for supercapacitors [J]. J. Mater. Chem. A, 2013, 1, 3454-3462.

[14] J. S. Wu, X. H. Rui, G. K. Long, W. Q. Chen, Q. Y. Yan, Q. C. Zhang. Pushing up lithium storage through nanostructured polyazaacene analogues as anode [J]. Angew. Chem. 2015, 127: $7462-7466$

[15] K. Gopalaiah, A. Saini, S. N. Chandrudu, D. C. Rao, H. Yadav, B. Kumar. Copper-catalyzed aerobic oxidative coupling of o-phenylenediamines with 2-aryl/heteroarylethylamines: direct access to construct quinoxalines [J]. Org. Biomol. Chem., 2017, 15: 2259-2268.

[16] D. Asakura, M. Okubo, Y. Mizuno, T. Kudo, H. Zhou, K. Ikedo, T. Mizokawa, A. Okazawa, N. Kojima. Fabrication of a cyanide-bridged coordination polymer electrode for enhanced electrochemical ion storage ability [J]. J. Phys. Chem. C 2012, 116: 8364-8369.

[17] L. Shen, Z. Wang, L. Chen. Prussian blues as a cathode material for lithium ion batteries [J]. Chem.- Eur. J. 2014, 20: 12559-12562.

[18] X. J. Wang, F. Krumeich, R. Nesper. Nanocomposite of manganese ferrocyanide and graphene: A promising cathode material for rechargeable lithium ion batteries [J]. Electrochem. Commun. 2013, 34: 246-249.
[19] M. Okubo, D. Asakura, Y. Mizuno, J.-D. Kim, T. Mizokawa, T. Kudo, I. Honma. Switching redox-active sites by valence tautomerism in prussian blue analogues $\mathrm{A}_{\mathrm{x}} \mathrm{Mn}_{\mathrm{y}}\left[\mathrm{Fe}(\mathrm{CN})_{6}\right] \cdot \mathrm{nH}_{2} \mathrm{O}(\mathrm{A}: \mathrm{K}, \mathrm{Rb})$ : Robust frameworks for reversible Li storage[J]. J. Phys. Chem. Lett. 2010, 1: 2063-2071.

[20] Y. Kurihara, T. Matsuda, Y. Moritomo. Structural properties of manganese hexacyanoferrates against Li concentration[J]. Jpn. J. Appl. Phys. 2013, 52: 017301.

[21] M. Okubo, I. Honma. Ternary metal prussian blue analogue nanoparticles as cathode materials for $\mathrm{Li}$-ion batteries[J]. Dalton Trans. 2013, 42: 15881-15884.

[22] M. Omarova, A. Koishybay, N. Yesibolati, A. Mentbayeva, N. Umirov, K. Ismailov, D. Adair, M. R. Babaa, I. Kurmanbayeva, Z. Bakenov. Nickel hexacyanoferrate nanoparticles as a low cost cathode material for lithium-ion batteries[J]. Electrochim. Acta 2015, 184: 58-63.

[23] M. H. Wong, Z. Zhang, X. Yang, X. Chen, J. Y. Ying. One-pot in situ redox synthesisof hexacyanoferrate/ conductive polymer hybrids as lithium-ion battery cathodes[J]. Chem. Commun. 2015, 51: 13674-13677.

[24] M. Xie, Y. X Huang, M. H. Xu, R. J. Chen, X. X. Zhang, L. $\mathrm{Li}, \mathrm{F}$. Wu. Sodium titanium hexacyanoferrate as an environmentally friendly and low-cost cathode material for sodium-ion batteries [J]. J. Power Sources 2016, 302: 7-12.

[25] Y. Z Jiang, S. H. Yu, B. Q. Wang, Y. Li, W. P. Sun, Y. H. Lu, M. Yan, B. Song, S. X. Dou. Prussian blue@C composite as an ultrahigh-rate and long-life sodium-ion battery cathode [J]. Adv. Funct. Mater. 2016, 26: 5315-5321.

[26] Y. You, X. Q. Yu, Y. X. Yin, K. W. Nam, Y. G. Guo. Sodium iron hexacyanoferrate with high $\mathrm{Na}$ content as a Na-rich cathode material for Na-ion batteries [J]. Nano. Res. 2015, 8(1): $117-128$.

[27] M. Xie, M. H. Xu, Y. X. Huang, R. J. Chen, X. X. Zhang, L. $\mathrm{Li}, \mathrm{F}$. Wu. $\mathrm{Na}_{2} \mathrm{Ni}_{\mathrm{x}} \mathrm{Co}_{1-\mathrm{x}} \mathrm{Fe}(\mathrm{CN})_{6}$ : A class of prussian blue analogs with transition metal elements as cathode materials for sodium ion batteries [J]. Electrochem. Commun. 2015, 59: 91-94.

[28] J. Song, L. Wang, Y. H. Lu, J. Liu, B. K. Guo, P. H. Xiao, J. J. Lee, X. Q. Yang, G. Henkelman, J. B. Goodenough. Removal of interstitial $\mathrm{H}_{2} \mathrm{O}$ in hexacyanometallates for a superior cathode of a sodium-ion battery [J]. J. Am. Chem. Soc. 2015, 137: $2658-2664$.

[29] M. H. Wong, Z. X. Zhang, X. F. Yang, X. J. Chen, J. Y. Ying. One-pot in situ redox synthesis of hexacyanoferrate/conductive polymer hybrids as lithium-ion battery cathodes [J]. Chem. Commun., 2015, 51: 13674-13677. 